\title{
Mapping electrical crosstalk in pixelated sensor arrays
}

S. Seshadri, D. M Cole, B. R. Hancock, R. M. Smith

S. Seshadri, D. M Cole, B. R. Hancock, R. M. Smith, "Mapping electrical crosstalk in pixelated sensor arrays," Proc. SPIE 7021, High Energy, Optical, and Infrared Detectors for Astronomy III, 702104 (22 July 2008); doi: $10.1117 / 12.790150$

SPIE Event: SPIE Astronomical Telescopes + Instrumentation, 2008, Marseille, France 


\title{
Mapping electrical crosstalk in pixelated sensor arrays
}

\author{
S. Seshadri ${ }^{\mathrm{a}}$, D.M. Cole ${ }^{\mathrm{a}}$, B.R. Hancock ${ }^{\mathrm{a}}$, R.M. Smith $^{\mathrm{b}}$ \\ a Jet Propulsion Laboratory, California Institute of Technology, MS 300-315, 4800 Oak Grove Drive, \\ Pasadena, CA USA 91109 \\ ${ }^{b}$ Roger M. Smith California Institute of Technology, MC 105-24, 1200 E. California Blvd, \\ Pasadena, CA USA 91125
}

\begin{abstract}
Electronic coupling effects such as Inter-Pixel Capacitance (IPC) affect the quantitative interpretation of image data from CMOS, hybrid visible and infrared imagers alike. Existing methods of characterizing IPC do not provide a map of the spatial variation of IPC over all pixels. We demonstrate a deterministic method that provides a direct quantitative map of the crosstalk across an imager. The approach requires only the ability to reset single pixels to an arbitrary voltage, different from the rest of the imager. No illumination source is required. Mapping IPC independently for each pixel is also made practical by the greater $\mathrm{S} / \mathrm{N}$ ratio achievable for an electrical stimulus than for an optical stimulus, which is subject to both Poisson statistics and diffusion effects of photo-generated charge. The data we present illustrates a more complex picture of IPC in Teledyne $\mathrm{HgCdTe}$ and $\mathrm{HyViSi}$ focal plane arrays than is presently understood, including the presence of a newly discovered, long range IPC in the HyViSi FPA that extends tens of pixels in distance, likely stemming from extended field effects in the fully depleted substrate. The sensitivity of the measurement approach has been shown to be good enough to distinguish spatial structure in IPC of the order of $0.1 \%$.
\end{abstract}

Keywords: inter pixel capacitance, electrical coupling, crosstalk, imager, focal plane array, detector, visible, infrared

\section{INTRODUCTION}

Many industrial, scientific and commercial applications that use electronic imagers rely on accurate knowledge of the quantitative information in image data. A small subset of relevant examples include color balance correction in imaging cameras, shape-measurement in industrial robotic and/or quality-control applications, Point Spread Function (PSF) determination in scientific instruments and identification of spectral lines and calculation of absorption and transmission strengths and linewidths in spectrometers. However, the quantitative information obtained from these imagers is often corrupted by artifacts from the image acquisition process.

For astronomy applications, spatial variations in the Point Spread Function of an imager has to be understood for (a) photometry to accurately subtract the background, (b) astrometry to accurately calculate centroids and (c) shape measurement such as weak lensing. For these and other scientific applications the required photometric precision can reach sub-percentage level accuracy. Emerging applications, such as weak lensing, require shape determination at mean accuracies of $<0.1 \%$. ${ }^{1}$ Such precision measurements require a re-evaluation of imager characteristics at the pixel level and/or development of new characterization methods. This is most critical for imaging surveys where the PSF is undersampled to maximize field of view and signal per pixel. Potential causes of distortions that must be measured for every pixel include: QE variations within pixel, charge diffusion, gain, linearity, crosstalk, as well as charge trapping effects such as Charge Transfer Efficiency (CTE) for CCDs and persistence for hybrid \& CMOS devices. The last issue is addressed elsewhere in these proceedings. ${ }^{2} \mathrm{We}$ focus, herein, on measuring electronic crosstalk which is dominated by Inter-Pixel Capacitance (IPC), as understanding its spatial variation is a necessary step in developing pixel-level characterization of the remaining sources of distortion.

IPC is the presence of electronic coupling between pixels in an imager. The existence of IPC was first determined using autocorrelation calculations of shot noise in pixels to explain inconsistencies in calculated QE from then-existing gain measurements generated from the shot noise measurements. ${ }^{3,4}$ This stochastic method provides a measure of coupling as

High Energy, Optical, and Infrared Detectors for Astronomy III, edited by David A. Dorn, Andrew D. Holland, Proc. of SPIE Vol. 7021, 702104, (2008) · 0277-786X/08/\$18 · doi: 10.1117/12.790150 
an average value across an imager. A subsequent approach used flat field illumination, followed by resetting of individual pixels of an imager. ${ }^{5}$ However, this method is limited in sensitivity by the shot noise in illumination and in spatial sampling by the practicalities of resetting pixels one at a time, relying on the window readout mode for the Teledyne H2RG multiplexor, which makes this method prohibitively slow. Additionally, this approach requires a reillumination of the Focal Plane Array (FPA) for each pixel tested. Thus, this latter approach can provide localized IPC measurements at a few pixels in an imager, but cannot practically provide a detailed map of the electronic coupling effects on all pixel elements of a focal plane array. Although this method could be modified to resemble the one we will describe, in order to overcome the limitation due to the shot noise in the illumination, we consider it preferable to eliminate the reliance on a light source and to adopt a technique which is able to electrically emulate the illumination patterns typical of the applications (point sources on a dark background, rather than dark spots in a flat field): Electrical signals can also be applied more rapidly, be controlled more precisely, and have either polarity.

We present a deterministic methodology to quantify spatial variability (extent and magnitude) in IPC. The procedure consists of resetting all of the pixels in the entire focal plane array to one voltage level, followed by resets of a grid of individually selected pixels to a second voltage level. Capacitive coupling will result in a signal in the adjacent pixels that can be detected by creating difference images from data taken before and after the second set of resets. Multiple sets of images can be used to beat down measurement noise. No illumination source is required. These changes allow us to sample the entire imager at sensitivities limited by the read noise rather than shot noise. Although there is an irreducibale floor to the noise on the level to which single pixels are reset, this level is measured after the reset. Also, the relevant measurement is the ratio of the change on the pixel which has been reset to the change on it neighbors, so this source of noise divides out. The measurements are, therefore, both direct and fully deterministic. The only requirement is that the FPA timing and control electronics has the ability to reset individual pixels.

\section{EXPERIMENTAL METHOD}

We demonstrate our approach using Teledyne Imaging Systems 2k x 2k, 1.7 um HgCdTe (HCT) infrared (IR) and $1 \mathrm{k} \mathrm{x}$ $1 \mathrm{k}$, HyViSi visible FPAs, hybridized to H2RG and H1RG multiplexors, respectively. The experiments were made possible using the Horizontal Enhanced Clocking (HEC) mode of the H1RG/H2RG multiplexors. This mode is provided by Teledyne to allow for sub-row time exposures. Our modifications explored various methods to clock through the focal planes and to control the grid of pixels that are reset in each experiment to create the IPC map. The spacing of the reset pixels is determined by the number of nearest neighbors over which the coupling coefficient is expected. Thus, one can use a spacing of as few as 3 pixels if only nearest neighbor or nearest diagonal neighbor coupling is present.
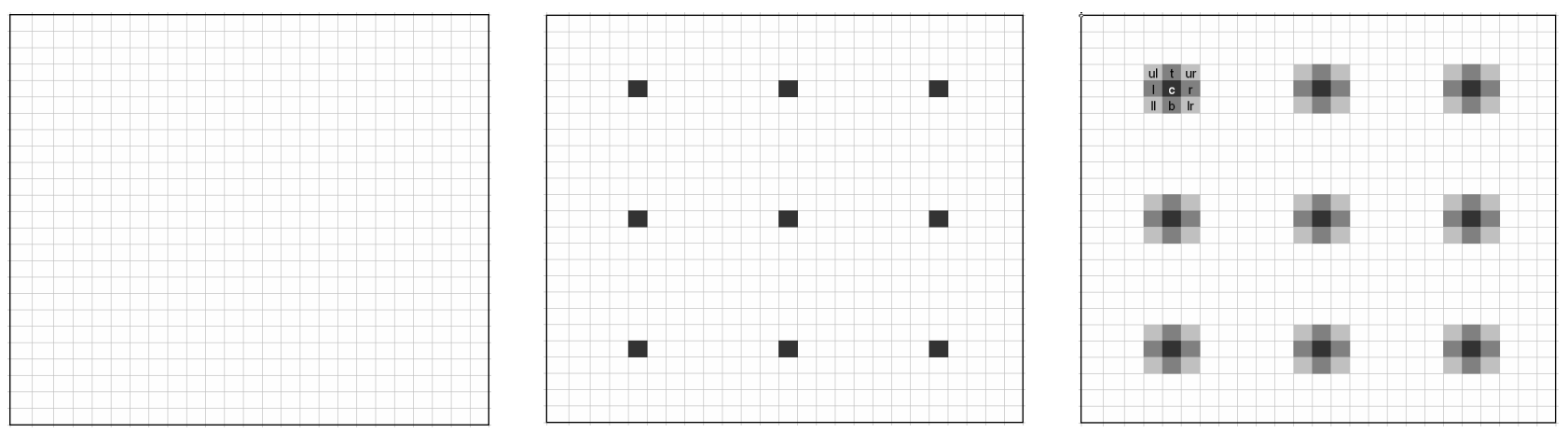

Figure 1: Cartoon illustrating the IPC mapping experiment: (a) A full field reset of the FPA at a given reset voltage. (b) A GRP frame in which a grid of pixels are reset to a different voltage, individually (but simultaneously in each output channel of the FPA), at a definable row and column spacing. (c) Difference image created by subtracting a post-reset image from the pre-reset image. The topleft section of the cartoon illustrates the nomenclature used in analyzing the data: $\mathrm{c}=$ center (reset) pixel; The sequence: $1=$ left, $\mathrm{r}=\mathrm{right}$, $\mathrm{t}=$ top $\& \mathrm{~b}=$ bottom represent the four $1^{\text {st }}$ nearest neighbor pixels; The sequence: $u l=$ upper left, ur=upper right, $1 \mathrm{l}=$ lower left $\&$ lr $=$ lower right represent the four $2^{\text {nd }}$ nearest neighbors or nearest diagonal neighbor pixels. 
Figure 1 schematically illustrates the experimental data sequence. A set of 4 CDS frames are taken prior to the start of the experimental sequence to ensure that the FPAs are in steady state operation. This is done to prevent any transient thermal effects caused by clocking through the FPA from affecting the data. We then obtain a normal reset frame at a single reset voltage and take a series of minimum-exposure, sample-up-the-ramp (SUTR) frames ( 8 , in our experiments). A grid of pixels is then reset to a different voltage, individually, but simultaneously in each output channel of the FPA, for a specified row and column spacing. We call this the GRP (Grid of Reset Pixels) frame. Another series of minimumexposure, SUTR frames ( 8 , in our experiments) is then taken without resetting any of the pixels to establish the stability of this post-GRP reset frames. Data for constructing the full IPC map is obtained by repeating this sequence, varying the row and column offsets until coverage has been achieved for all pixels. In our mapping demonstration experiment we used either an 8-pixel or 7-pixel pitch between reset pixels in both the row and column dimension. A 7-pixel pitch required a sequence of 49 experiments to obtain the full FPA map. The data volume of the raw images was (49 experiments x 25 frames/experiment x 4 Mbyte/frame) $>5$ Gbytes, tripling to $>15$ Gbytes after completion of the data analysis (assuming one wants to preserve the intermediate images that are created). The experiment can be performed in one day.

The 8-frame sequences before and after the GRP frame allow determination of the strength of image artifacts such as light leakage, dark current, drift, etc.. In the absence of these effects, the difference images of frames before and after frames allow direct determination of the voltage-to-voltage coupling factors for the reset pixels and their neighbors. In practice, we use the second frame of the post-GRP 8-frame sequence to avoid issues we have historically seen in the image immediately succeeding the post-GRP frame.

All exposures were done at the minimum frame time with the HyViSI and HCT FPAs in 16- and 32-channel output mode. While the $2 \mathrm{k}$ x $2 \mathrm{k}$ HCT FPA was setup in the 32 channel output mode, our data acquisition system was only set up to read out the first 16 channels. Thus, the images of the HCT FPA contain only half the columns and channels in the array. This distinction is an experimental detail that does not affect the general applicability of the method. Measurements on the HCT and HyViSi FPAs were made at 140K and 170K, respectively. The bias on the HyViSi was $10 \mathrm{~V}$. A voltage of $0.5 \mathrm{~V}$ was used to reset all the pixels in the FPA. The grid of reset pixels used a voltage of $1.0 \mathrm{~V}$ for the HCT FPA and $0.95 \mathrm{~V}$ for the HyViSi FPA. Thus, the voltage step for the IPC calculation was $0.45 \mathrm{~V}$ for the HCT FPA and $0.5 \mathrm{~V}$ for the HyViSi FPA. The enhanced clocking mode of the H1RG and H2RG multiplexors allows two reads of each pixel within a row time. The H1RG and H2RG also offers modes to alter the horizontal and vertical scan direction of the readout and to prescribe the clock edges on which pixels can be read out. We varied which pixel read we used and the readout sequences in our experimental matrix to differentiate between clocking related artifacts (which one should nevertheless be aware of) and intrinsic spatial variations in the IPC for the FPAs. Finally, the two methods we used to create the x-offsets for the reset pixels required us to violate rules for creating the timing offsets between the first and second pixel reads. These appear to introduce artifacts into the IPC maps. We have identified a third clocking scheme that should get around this violation, but have not yet tested it.

Three kinds of images are shown in this paper: (1) raw images, which are simply digitized (deinterleaved, since the clocking mode allows two reads of each pixel) outputs of the FPA readouts; (2) difference images of two raw, deinterleaved images; and (3) IPC maps constructed by processing the difference images, typically by subtracting a background and taking the ratio of the neighboring pixel values to the corresponding center (reset) pixel value. Images shown in this paper contain a sequence of 6 pixels at the beginning of each channel that were not mapped, simply because our code did not access these pixels. In a different implementation we could have reduced this number to the first two pixels of each channel. Using this approach and operating the H1RG and H2RG multiplexors (muxes) in single output mode would enable access to all the operating pixels in the FPA, since the two muxes have an annulus of four reference pixels. We did not focus on implementing this refinement in our timing code. Therefore, the images shown in the paper will contain a set of columnar banded features at the start of each channel, corresponding to these neglected pixels. 


\section{RESULTS AND DISCUSSION}

Figure 2 shows actual images from the HCT FPA, corresponding to the cartoons in Figure 1. The IPC is clearly distinguishable in the difference measurements, despite the fact that, as one shall see below, it is of a fairly small value. It is easily seen that the nearest neighbors are strongly affected. Some residual IPC in the nearest diagonals is also visible for the HCT FPA.

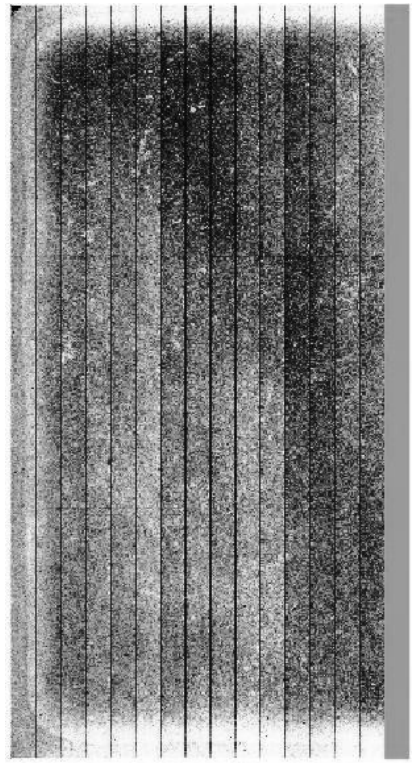

(a)

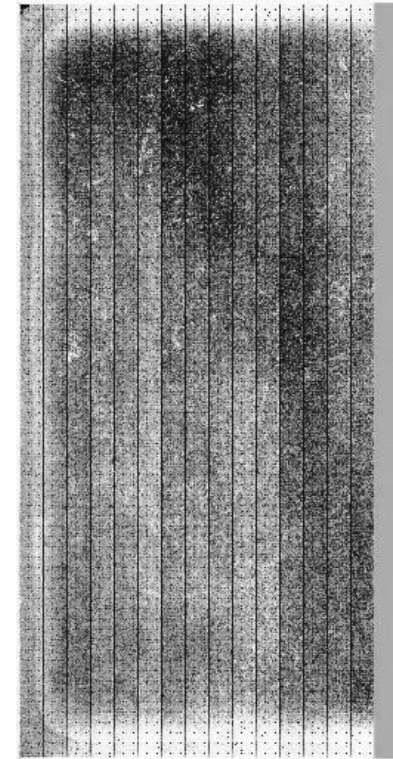

(b)

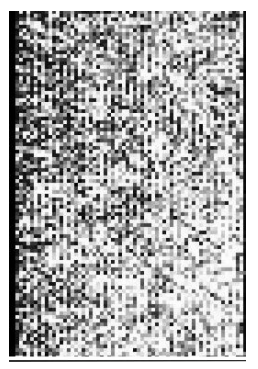

(c)

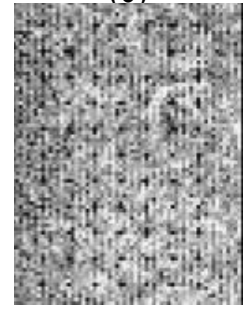

(d)

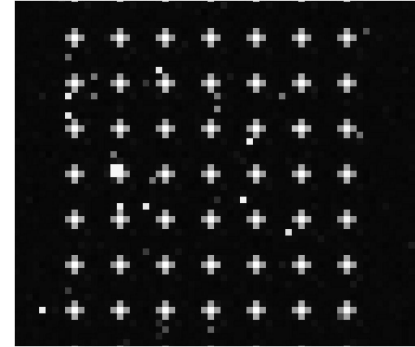

(e)

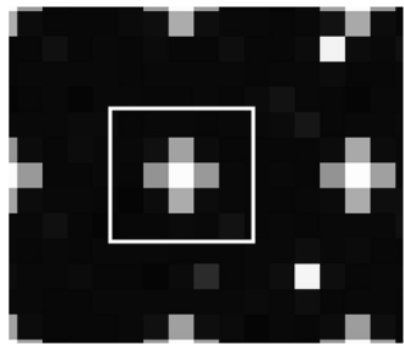

(f)

Figure 2: Representative images for the first 16 of 32 channels of a $2 \mathrm{k} x$ 2k a Teledyne $\mathrm{HgCdTe} / \mathrm{H} 2 \mathrm{RG}$ corresponding to (a) before and (b) after the GRP frame, as in Figure 1a and b. A magnified view spanning a single channel of the (c) before and (d) after images in a \& b. (e) Difference image of the single channel, formed by subtracting the two images. (f) A magnified view of the IPC around one reset pixel. The white square defines a $5 \times 5$ pixel region around the reset pixel inside of which we calculated IPC parameters.

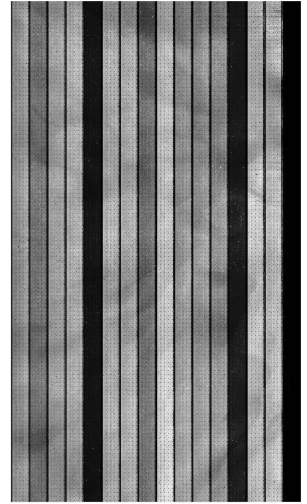

center (reset)

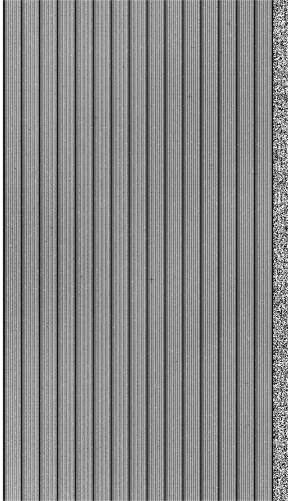

Left

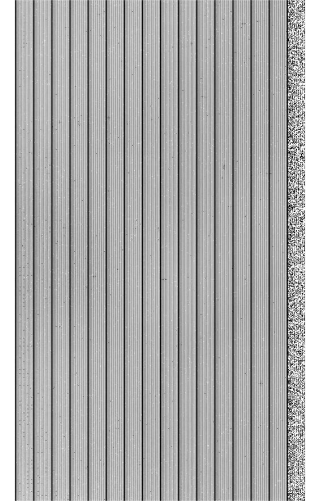

right

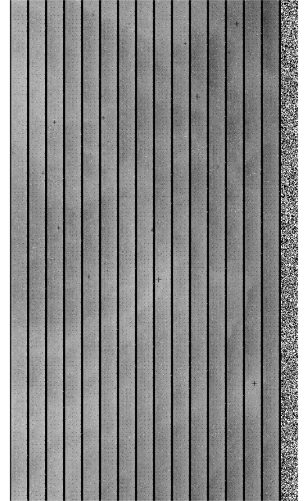

top

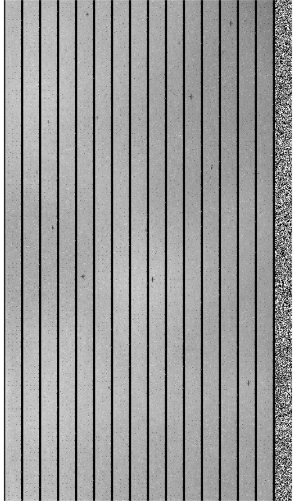

bottom

Figure 3: IPC maps, as seen in figure $2 \mathrm{e}$ and $2 \mathrm{f}$, from 16 of 32 channels of $2 \mathrm{k}$ x $2 \mathrm{k}$ HCT/H2RG imager $(>3.4 \mathrm{MPixels}$ of 4 MPixels, if all 32 channels had been sampled). The reset map represents the difference in reset voltage of each pixel from its full frame reset value. This difference voltage is what is used to determine the IPC in the neighboring pixels. The remaining four images correspond to IPC maps of each sampled pixel in the FPA when it was the left, right, top and bottom nearest neighbor to the pixels that were reset. Each channel in the image is 64 pixels wide, with its start being defined by the black columns in each image, which correspond to (6) pixels at the beginning of each channel that were not sampled in the creation of this map. 

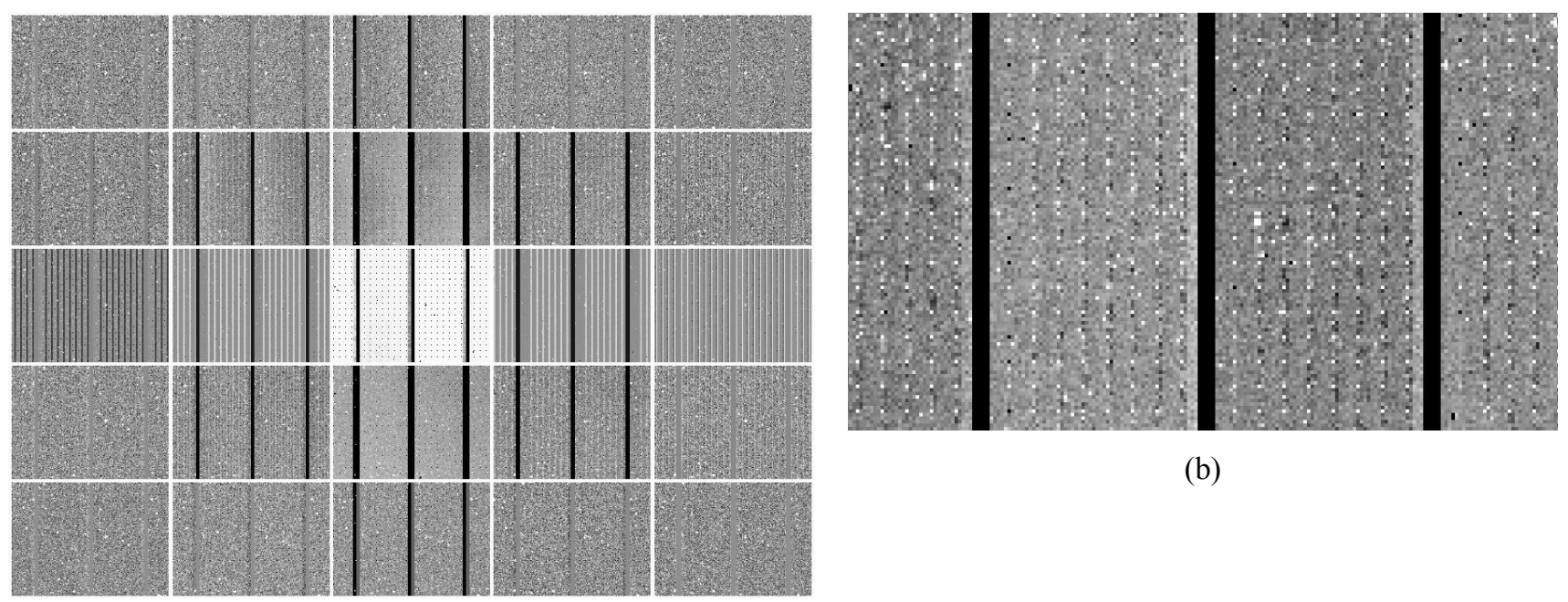

(b)

(a)

Figure 4: (a) A set of 25 image maps corresponding to a set of pixels from a section near the center of a $2 \mathrm{k} x 2 \mathrm{k}$ HCT/H2RG imager. The center (bright) image corresponds to a map of the difference in reset voltage values for each pixel in this section when it was the reset pixel. The eight images immediately surrounding the center image correspond to IPC maps (voltage difference of each pixel when it was the $1^{\text {st }}$ and $2^{\text {nd }}$-nearest neighbor pixel, divided by the voltage difference of the corresponding reset pixel). The remaining 16 images that make up the outer annulus of images represent IPC maps corresponding to when each pixel in this section of the focal plane was the $3^{\text {rd }}, 4^{\text {th }}$ and $5^{\text {th }}$ nearest neighbor to the reset pixels. (b) Right nearest neighbor IPC image created after an additional subtraction step to remove row and column averages from the difference images does not contain the clocking-induced stripping artifact present in the corresponding IPC map in (a).

Figure 3 and Figure 4 illustrate IPC maps for the HCT FPA. The banding of channels in the reset difference image indicates the presence of some variation in gain from channel to channel. It is interesting to note that the top and bottom IPC maps replicate the diffuse structure in the reset map, even after constructing the IPC map by taking the ratio of the neighboring pixel voltage differences to the center (reset) pixel difference value. In contrast, such structure is absent in the left and right IPC map. However, these, and the nearest diagonal neighbors, harbor a residual stripping that is not present in the top and bottom IPC maps. This stripping is more visible in the magnified images in Figure 4.The black columns in the center image and in the IPC maps of the $1^{\text {st }}, 2^{\text {nd }}$ and $3^{\text {rd }}$ nearest neighbors, as well as the thick, solid vertical banding in the remaining IPC maps, represent the (6) pixels at the beginning of each channel that were not sampled in the creation of this map. The random noise in the farthest (i.e. $5^{\text {th }}$ nearest neighbor) IPC map image is featureless, indicating the absence of any artifacts in the creation of the map. However, the additional columnar features within each channel in the $1^{\text {st }}, 2^{\text {nd }}, 3^{\text {rd }}$ and $4^{\text {th }}$ nearest neighbor maps represent artifacts of a known violation of the horizontal enhanced clocking mode timing rule. This artifact, for which we have devised, but not yet tested a work around, can also be largely removed by subtracting the column average difference voltage value, as shown in Figure $4 \mathrm{~b}$ ).

Figure 5 illustrates a single difference image for the HyViSi FPA. In this experiment, the pixel grid was confined to a region comprising most of the center of the FPA, but did not extend to many rows on either the top or the bottom of the FPA. It is clear from these images that, in addition to the "normal' IPC found between adjacent pixels in the HCT, there is a second, much broader effect that occurs on the scale of up to 20 pixels. These features are quantified later, in the histograms in Figure 11, which illustrate the IPC distribution of the HCT and HyViSi FPAs. The imager average nearest neighbor IPC of the HCT and HyViSi FPAs are seen to be $\sim 1.5 \%$ and $4.5 \%$, respectively. The nearest diagonal IPC of the two focal planes is seen to be $\sim 0.1 \%$ and $1.8 \%$, respectively. Finally, the background IPC is seen to be 0 and $1.2 \%$, respectively. Since the HyViSi is a high-IPC device, it is no surprise that the nearest diagonal of the HyViSi has a higher average IPC than the nearest neighbor for the HCT FPA. However, the non-zero background was not anticipated and fails the common understanding of the physical mechanism of IPC in these focal planes as a coupling of nearest neighbor pixels. We haven't yet conducted tests to confirm the cause of this effect. But it's large extent and specificity to the HyViSi focal plane precludes the source being in the multiplexor. Unlike HCT, HyViSi FPA is fully depleted by a large backside bias voltage. Thus, it is reasonably conceivable that a change in voltage of an individual pixel leads to a 


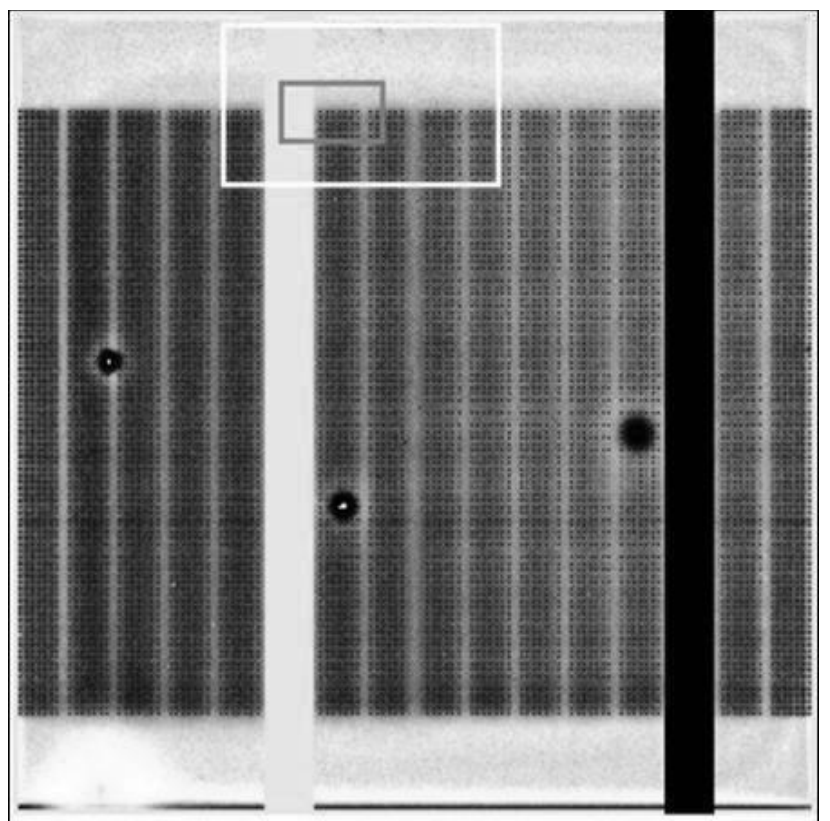

(a)

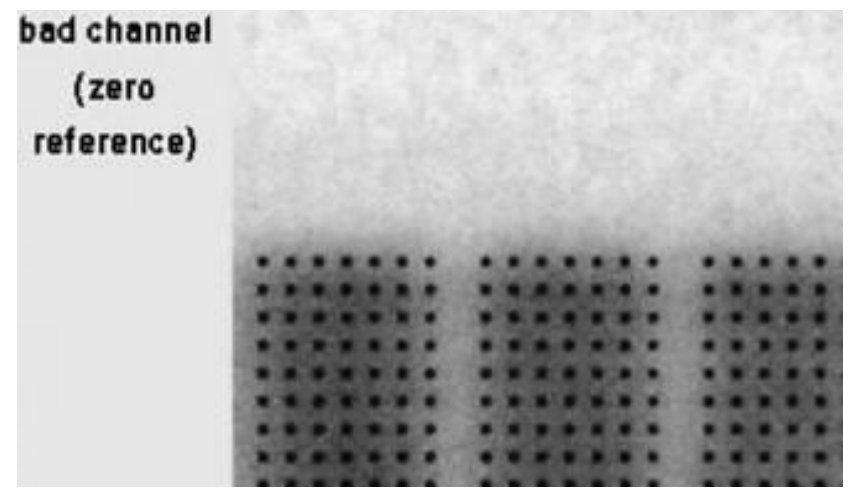

(b)

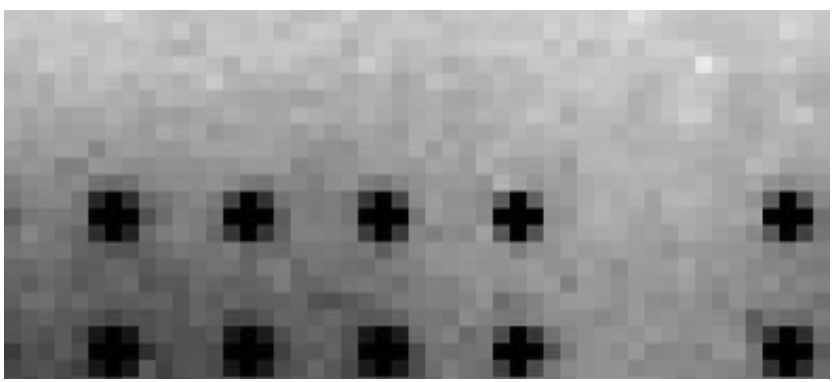

(c)

Figure 5:(a) Full difference images from a Teledyne 1kx1k HyViSi/H1RG FPA. Expanded view of the regions defined by the (b) white and (c) gray boxes at the top of the image in (a). The difference image is of opposite polarity to that for the HCT FPA in figure $2 \mathrm{e}$ and figure $2 \mathrm{f}$. Two channels $(6 \& 14)$ are not operating properly. IPC is noticeable for the nearest neighbors and nearest diagonal neighbors with an additional diffuse background, compared to the pixel values at the edges of the FPA, where no pixels were reset. This background is not explained by conventional understanding of IPC.

slight realignment of electric field lines (to an extent that depends on the physical pixel layout), modifying depletion region edges, and hence, coupling capacitances of pixels over greater distances than would occur non-fully depleted FPAs such as the HCT. This observation may be a non-destructive manifestation of anecdotal, unpublished data on the HyViSi FPA, currently being referred to as "persistent persistence," in which, under certain conditions, the baseline voltage of a pixel is irreversibly altered after highly oversaturating the FPA.

We now examine these features in more detail. There are a series of questions about accuracies and artifacts of the measurement process that we need to answer before coming to conclusions about the real spatial variability of the IPC. The first of these is the noise level and (spatial and temporal) repeatability of an individual measurement.

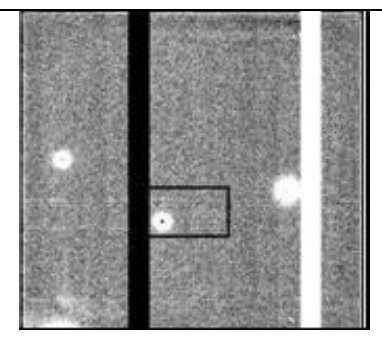

(a)

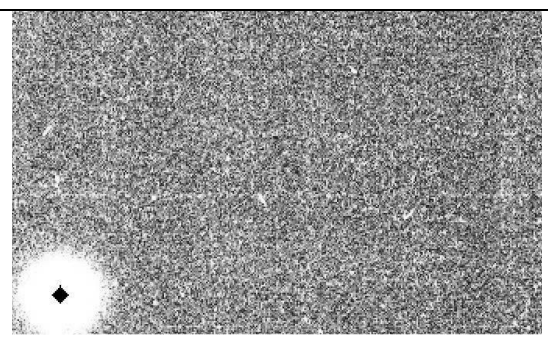

(b)

Figure 6: (a) Noise image illustrating the temporal variation of a region of the HyViSi FPA obtained by repeating the IPC experiment, 32 times, resetting the same reset pixel grid in each experiment. The noise image is seen to be essentially flat, with the exception of the two non-working channels and three defect regions. (b) Expanded view of the region defined by the rectangular box in (a).

We have addressed the temporal question by repeating this experiment (on the same grid of reset pixels) 32 times using the HyViSi FPA. (i.e. the frame sequence illustrated in Figure 1 was repeated 32 times with resets occurring on the same pixels for each of the repeat experiments). The 32 difference images formed from the raw data for each of the 32-experiments were averaged. The temporal noise image from this experiment 
is featureless, except for the bad channels and a couple of hot spots around defects, as shown in Figure 6. The square root of the average of the temporal variance of the FPA was 42 ADU.

Figure 7 is a correlation plot that compares difference voltages for the center (reset), nearest neighbor and background pixels, obtained from a single IPC experiment, against that obtained from an average of 32 experiments. Table 1 summarizes the results of linear least square fits for the center and each neighbor pixel plotted in Figure 7. The tight distribution and linearity of the data in Figure 7 indicates that the experiments were highly repeatable, that signal variations are statistically significant and truly represent structure in the spatial variance of the IPC of this FPA. The standard deviation of the difference of the signal level in the nearest neighbor from the least square fit curve is a measure of the temporal variance of the IPC. The roughly uniform $25 \mathrm{ADU}$ deviation for all the nearest neighbor values is dominated by read noise, absent drift that must be present in the temporal noise data (because of its higher standard deviation).

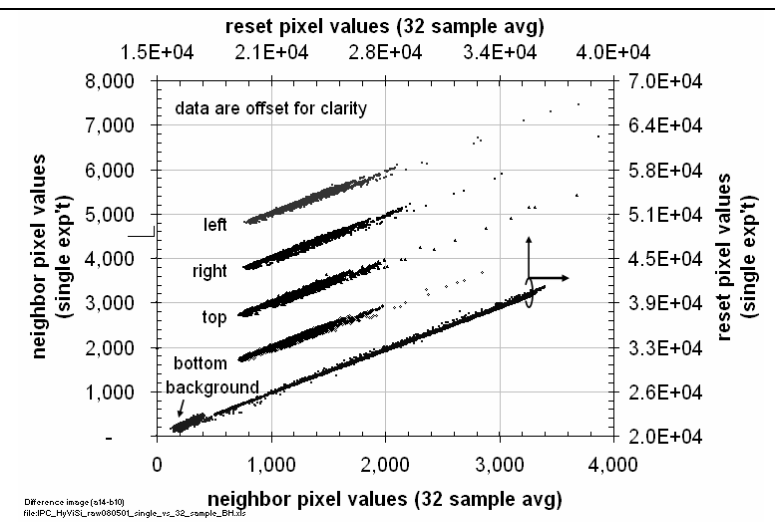

Figure 7: Correlation plot of the voltage values (in ADU) for a single experiment compared to that for a 32-sample average for the HyViSi FPA. The response is tightly distributed and linear, as expected.

Figure 8a compares the correlation of the neighbor pixel difference voltages shown in Figure 7 against the center (reset) pixel difference voltage. Three features are worth noting from the figure. First, the slope of the points in Figure 8a, which is indicative of the average IPC in the FPA appears to be constant for signal levels of all neighbor pixels up to $\sim 35,000 \mathrm{ADU}$, at which point the IPC increases dramatically. These high value pixels have been traced to be spatially isolated to channel 1 . It is not clear from these measurements alone, whether this is due to a real variation in IPC or a signal level effect. Second, in contrast with the HCT FPA (not shown), the HyViSi

Table 1: Summary statistics for the nearest neighbor pixels for a 32 sample average for the HyViSi FPA. This corresponds to the data in Figure 7.

\begin{tabular}{|l|r|r|r|}
\hline \# pts= 9502 & slope & intercept & stdev of err \\
\hline center & 0.969 & -389 & 116.1 \\
\hline top & 0.959 & -42 & 24.8 \\
\hline right & 0.970 & -26 & 25.0 \\
\hline bottom & 0.967 & -27 & 25.0 \\
\hline right & 0.979 & -13 & 25.3 \\
\hline background & 0.929 & -37 & 25.4 \\
\hline
\end{tabular}
background pixel is significantly affected by the reset pixels, with a different (though lower) IPC value and offset from those for the nearest neighbors. Third, the deviation from a fit to the data, illustrated for the right nearest neighbor in Figure $8 b$, represents the spatial variation of the IPC. It is not constant, but rather, increases with increasing signal.

Coupling between pixels is commonly assumed to scale with the driving signal. Thus, IPC is defined as the ratio of a neighbor pixel value to the center pixel value. The relative tightness of the correlation plot in Figure 8a and its near-zero intercept says that the assumption of linearity is reasonable for the HyViSi FPA. We are now in a position to calculate the IPC.

Careful examination of the curves in Figure 8a indicate the presence of two IPC distributions. This is most clearly seen in the error curve in Figure $8 \mathrm{~b}$ and in the histogram in Figure 8c, where one can see a shoulder in the single experiment data, which is revealed to be a second distribution in the 32-experiment average data. The origin of the secondary distribution is seen in the row cross section in Figure 9. A systematic variation is present across each channel on the HyViSi focal plane. This is caused by a newly observed, long-range IPC, being affected by the break in symmetry in (7pixel) pitch for GRP pixels within each channel and the larger pitch difference across channels (between the last pixel reset in each channel and the first reset pixel in the adjacent channel). Undulation in the column dimension and slope in the row dimension are also visible. The first and last channels of the FPA are also seen to deviate from the imager 


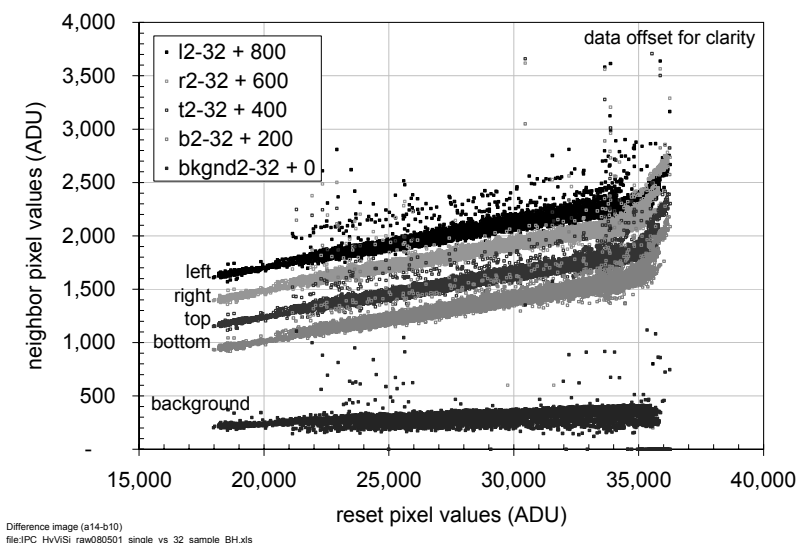

(a)

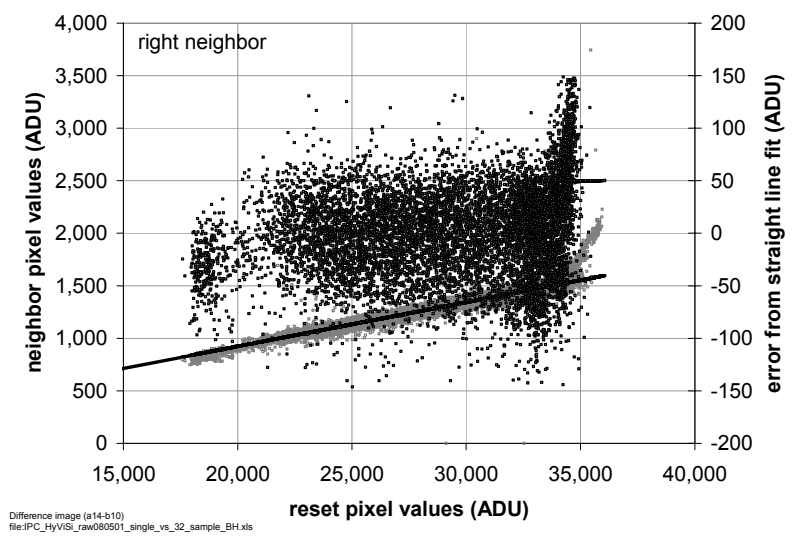

(b)

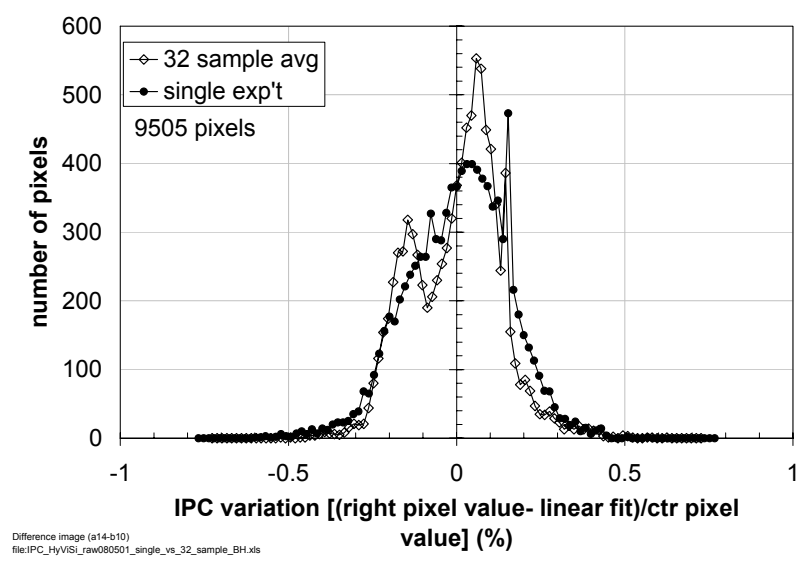

(c)

average, with a smaller bowing noticeable for intermediate channels. Finally, a very slight gradient in the column direction is seen in the IPC.

Figure 10 shows a very different row and column average pattern for the HCT to that seen for the HyViSi FPA. The

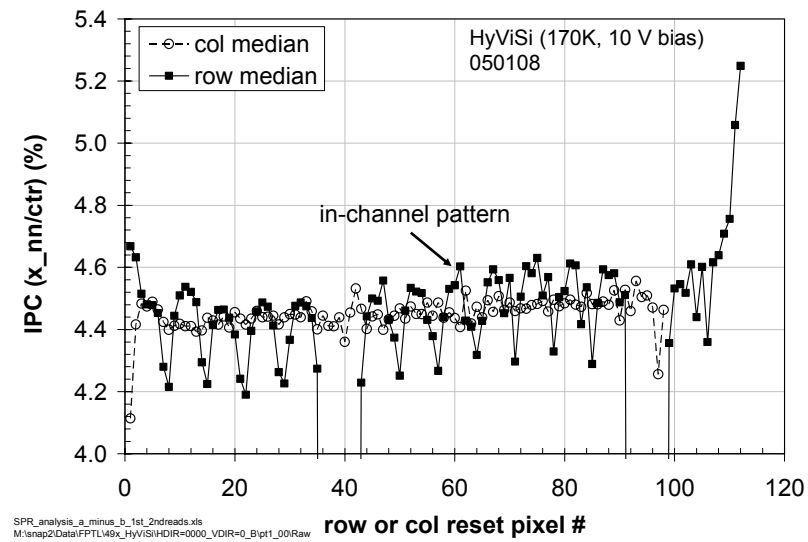

Figure 9: Row and column cross sections of the IPC of the HyViSi FPA.

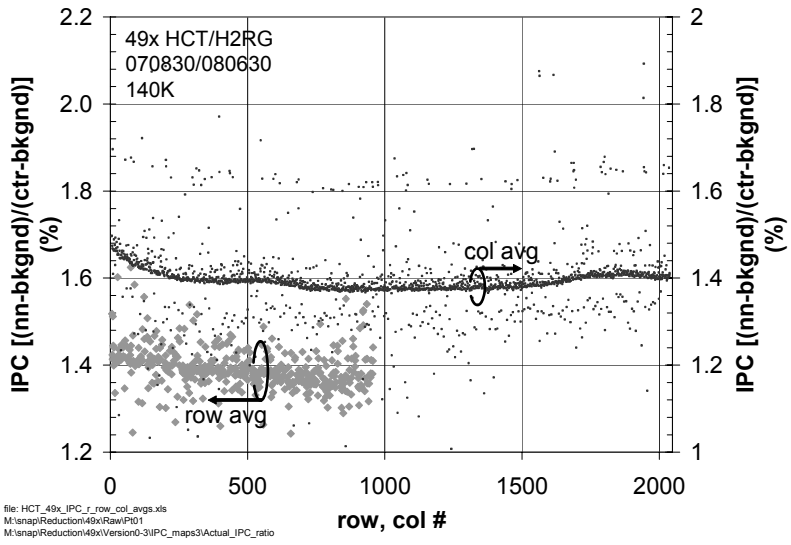

Figure 10: Row and column average IPC for one of the 49 experiments used to map the IPC of the HCT FPA. 
HCT does not have the in-channel IPC variation observed for the HyViSi. Undulations seen in this figure are the result of the diffuse structure visible in Figure 3 that represents the large-scale variation in IPC across the FPA. Note that we are measuring very slight $(\sim 0.1 \%)$ variations in IPC across the FPA.

Figure 11 illustrates distributions of IPC for the HCT and the HyViSi FPAs. Note that the background IPC of the HCT FPA is centered on zero, indicating the absence of residual effects. The mean (and Full Width at Half Maximum - i.e. FWHM) IPC for the nearest neighbor HCT FPA is between 1.4\% for the left and $1.55 \%(0.12 \%)$ for the bottom nearest neighbor. In comparison, Finger et. al. ${ }^{5}$ reported IPC from single pixels of upper $1.4 \%$, lower $1.5 \%$, left $1.8 \%$, right $1.7 \%$. They also noted that IPC of up to $2.5 \%$ were observed on other HCT arrays. Thus, the two sets of numbers are closely matched. They also show an asymmetry in IPC of nearest neighbors in the row and column direction, but the relative magnitudes are of opposite sense in the two instances. The next nearest diagonal neighbor IPC is $0.13 \%$ $(0.02 \%)$. Noise autocorrelation measurements for the HCT FPA, on the other hand, yield a value of $\sim 2 \%$ for the nearest neighbor IPC. This differs from both our results and those of Finger et. al. There is an outstanding question as to whether the $\sim 0.45 \%$ to $\sim 0.6 \%$ difference is real, owing to the differences in focal plane arrays, measurement methodology and/or the inclusion of charge diffusion effects in the autocorrelation measurements that is not present in these measurements.
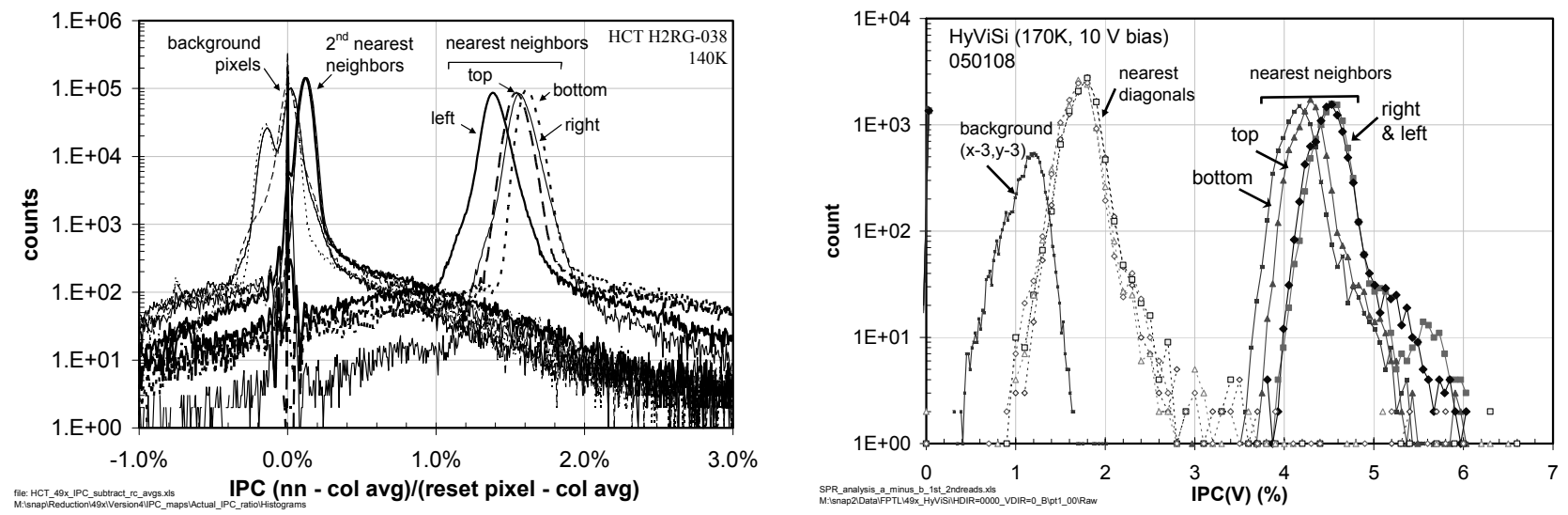

Figure 11: Log-linear histograms showing the variation of IPC of the (a) HCT and (b) HyViSi FPAs. Both histograms are from single experiment data and comprise a sample of $>1.9 \times 10^{6}$ pixels for the HCT and $>10^{3}$ pixels for the HyViSi.

The IPC of nearest neighbor pixels for the HyViSi FPA has a mean of $4.2 \%$ to $4.6 \%$ with a FWHM of $0.25 \%$ to $0.35 \%$. We cannot clearly separate spatial variation from read noise from this experiment because the standard deviation is the same order of magnitude. However, we can see the emergence of a slight shoulder on the low IPC side that is related to the spatial structure shown in Figure 8c and Figure 9. The IPC of the nearest diagonal neighbor pixels of the HyViSi FPA is a bit higher than the IPC of the nearest neighbor pixels on the HCT FPA. However, the IPC of the background pixels in the HyViSi FPA is NOT zero, as might be expected, but rather almost as large as the nearest neighbor IPC of the HCT FPA. This quantifies both the IPC variation corresponding to the shaded regions in Figure 5, in the region where the grid of pixels were reset, and the background difference voltage values shown in Figure 7 and in Figure 8c. In comparison, Finger et. al. ${ }^{5}$ report IPC of $8.2 \%, 8.8 \%, 10.2 \%, 8.45 \%$ for the upper, lower, left and right nearest neighbors, respectively. These values are considerably higher than what we have found. However, IPC in these devices is known to strongly depend on detector bias conditions. We could not find information on the bias voltages used in the other experiment. We cannot, therefore, make more quantitative comparisons of the two experimental results. In contrast, to the case for the HCT FPAs, however, Finger et.al. do state that PSFs calculated from autocorrelation results ${ }^{6}$ match those obtained from their single pixel results.

We explored the effect of clocking on these IPC distributions to try to understand the cause of variations in IPC between nearest neighbor pixels for the HyViSi FPA. Figure 12 illustrates the effect of changing the horizontal and vertical scan 
directions. Changing the horizontal clocking direction results in no change in IPC, indicating that the absence of clocking-induced artifacts on the baseline IPC. However, changing the vertical clocking direction does appear to flip the IPC value of the top and bottom nearest neighbor pixels at the level of $\sim 0.2 \%$ IPC, indicating that this small asymmetry is caused by clocking effects. Finally, there does appear to be an $\mathrm{x} / \mathrm{y}$ asymmetry in IPC of $\sim 0.4 \%$ between the top/bottom nearest neighbors and the left/right nearest neighbors for the HyViSi FPA. We have not yet analyzed similar experiments for the HCT FPA.

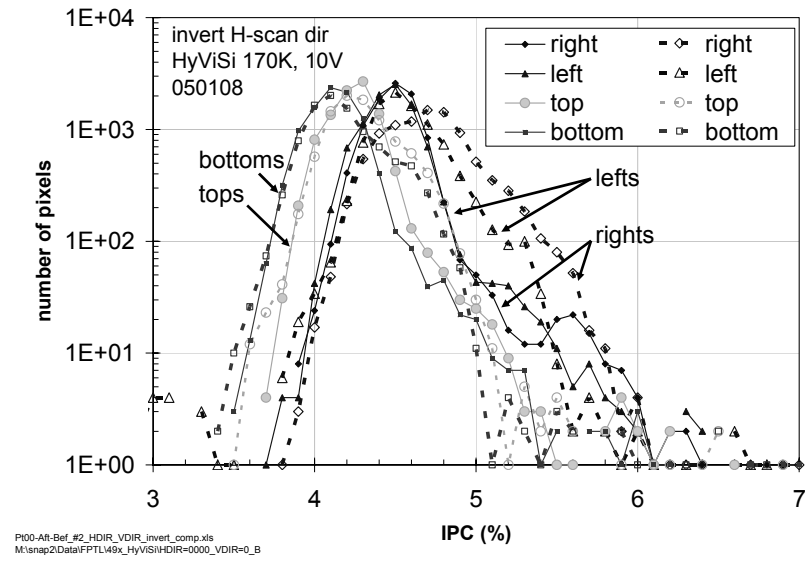

(a)

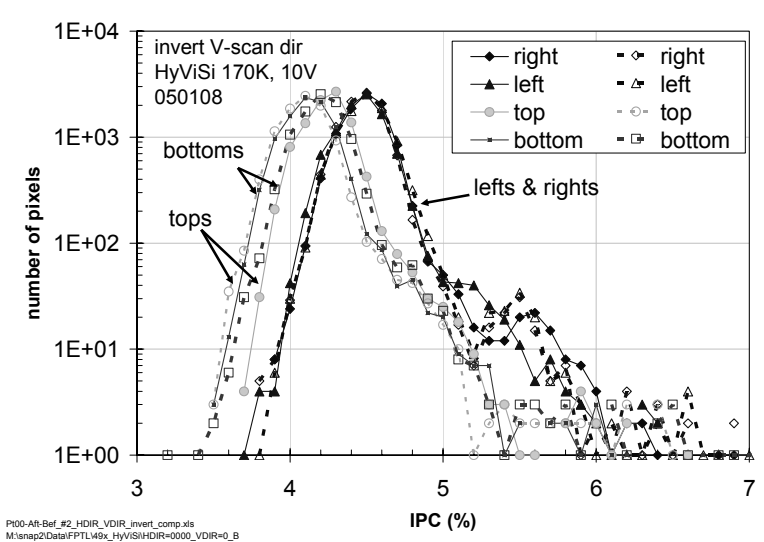

(b)

Figure 12: Variation of spatial distribution as a result of changing the (b) horizontal and (b) vertical clocking direction for the HyViSi FPA. The solid lines represent left-to-right or top-to-bottom clocking, while the dashed lines are right-to-left or bottom-totop clocking.

\section{CONCLUSIONS}

We have demonstrated a methodology to characterize spatial variations in the IPC of pixelated sensor arrays. The sensitivity of the measurement approach has been shown to be good enough to distinguish spatial structure of the order of $0.1 \%$, a sensitivity likely sufficient to evaluate impact of variations on weak lensing measurements and precision photometry requirements of many of the most scientifically challenging applications. This method is feasible in pixilated sensor arrays that provide the capability to electronically alter the signal value of individual pixels. We have also shown that spatial variation of IPC in Teledyne HyViSi and HCT focal planes is tightly distributed, but still possesses discernable spatial structure. The HyViSi also possesses an additional, long-range, IPC that extends over many pixels, likely stemming from extended field effects in the fully depleted substrate. Outstanding questions that remain include eliminating artifacts of clock direction and the order in which pixels are measured, evaluating the impact of variations on nodal capacitance (pixel gain) and developing \& assessing an appropriate correction algorithm.

\section{ACKNOWLEDGEMENTS}

This work was carried out at the Jet Propulsion Laboratory, California Institute of Technology, under a contract with the National Aeronautics and Space Administration and funded through the internal Science and Technology program. We also thank Jason Rhodes for his encouragement and funding support. The detector development and procurement was sponsored by the United States Department of Energy under contract No. DE-AC02-05CH11231. The HCT FPA was provided by Chris Bebek of the Lawrence Berkeley National Laboratory. The HyViSi FPA used was provided via David Crisp and Mark Schwochert of the NASA Orbiting Carbon Observatory mission. 


\section{REFERENCES}

${ }^{1}$ Rhodes, J., et. al. "The Stability of the Point Spread Function of the Advanced Camera for Surveys on the Hubble Space Telescope and Implications for Weak Gravitational Lensing" Astrophysical Journal Supplement Series, V172, p203-218. (2007), astro-ph/0702140v1.

${ }^{2}$ Smith, R.M., Zovodny, M. and Rahmer, G., "A theory for image persistence in HgCdTe photodiodes," and

"Calibration of image persistence in HgCdTe photodiodes" Proc. SPIE this volume, (2008).

${ }^{3}$ Moore, A.C., Ninkov, N. and Forrest, B. "QE Overestimation and Deterministic Crosstalk Resulting from Inter-pixel Capacitance", Optical Engineering (2003).

${ }^{4}$ Moore, A.C., Ninkov, N. and Forrest, B. "Interpixel capacitance in non-destructive focal plane arrays" Proc. SPIE, V5167 (2006).

${ }^{5}$ Finger, G., Dorn, R., Meyer, M., Mehrgan, L., Moorwood, A.F.M., and Stegmeier, J., "Interpixel capacitance in large format CMOS hybrid arrays", Proc. SPIE V6276, (2006).

${ }^{6}$ Dorn, R., Eschbaumer, S., Finger, G., Mehrgan, L., Meyer, M. and Stegmeier, J., “A CMOS Visible Silicon Imager hybridized to a Rockwell 2RG multiplexer as a new detector for ground based astronomy", Proc. SPIE 6276, (2006). 